\title{
Effect of Adding Facile Base Solution on Electrochemical Synthesis of Gold Nanoparticles
}

\author{
Pao-Hsun Huang, ${ }^{1}$ Po-Yen Lin, ${ }^{2}$ Po-Chen Lin, ${ }^{2}$ Shui-Yang Lien, ${ }^{3,4,5}$ \\ Po-Wen Sze, ${ }^{6}$ Chih-Chieh Kang, ${ }^{7}$ Na-Fu Wang, ${ }^{8}$ and Chien-Jung Huang ${ }^{2 *}$ \\ ${ }^{1}$ School of Information Engineering, Jimei University, \\ 183 Yinjiang Road, Jimei District, Xiamen, Fujian 361021, China \\ ${ }^{2}$ Department of Applied Physics, National University of Kaohsiung, Kaohsiung 81148, Taiwan \\ ${ }^{3}$ School of Opto-Electronic and Communication Engineering, Xiamen University of Technology, \\ Xiamen 361024, China \\ ${ }^{4}$ Department of Materials Science and Engineering, Da-Yeh University, Dacun, Changhua 51591, Taiwan \\ ${ }^{5}$ Fujian Key Laboratory of Optoelectronic Technology and Devices, Xiamen University of Technology, \\ Xiamen 361024, China \\ ${ }^{6}$ Department of Electrical Engineering, Kao Yuan University, Kaohsiung 82146, Taiwan \\ ${ }^{7}$ Department of Electro-Optical Engineering, Southern Taiwan University of Technology, \\ \#1 Nan-Tai St, Yung-Kang, Tainan 71005, Taiwan \\ ${ }^{8}$ Department of Electronic Engineering, Center for Environmental Toxin and Emerging-Contaminant Research, \\ Super Micro Mass Research \& Technology Center, Cheng Shiu University, \\ \#840, Chengcing Rd., Niaosong Dist., Kaohsiung 82146, Taiwan
}

(Received March 13, 2020; accepted September 23, 2020)

Keywords: electrochemical synthesis, gold nanoparticles, sodium hydroxide, condensation

In this research, the gold nanoparticles (GNPs) obtained by sono-electrochemical fabrication were prepared via the addition of a base solution, sodium hydroxide $(\mathrm{NaOH})$, into the growth solution. The effect of the amount of $\mathrm{NaOH}$ on the growth of GNPs was investigated. The growth solution was improved to blend collocated acetone, cetyltrimethylammonium bromide (CTAB), and $\mathrm{NaOH}$ for the evenness control of GNPs. The excessive condensation reaction resulting from acetone was inhibited by adding $70 \mu \mathrm{l}$ of $\mathrm{NaOH}$ to the growth solution to build a micelle template. Uniform and spherical GNPs with an average diameter of $25.5 \mathrm{~nm}$ and an absorption peak at $527 \mathrm{~nm}$ were obtained because the interaction between free electrons and hydroxyl groups loosened the micelle template. The cluster agglomeration and size distribution of GNPs were also improved according to transmission electron microscopy (TEM) observation. The results of this study can be applied to sensors to improve their sensitivity by adding more nanoparticles or enhancing the sensor signal.

\section{Introduction}

Over the past decade, there has been great interest in nanomaterials owing to their unique optical and magnetic properties. ${ }^{(1-7)}$ The synthesis of multiform nanoparticles (MNPs) when a noble bulk metal (gold, silver, or platinum) is reduced to nanometer size has been extensively investigated, ${ }^{(2)}$ leading to the exponential increase in the number of studies on their preparation

*Corresponding author: e-mail: chien@nuk.edu.tw https://doi.org/10.18494/SAM.2021.2859 
and applications. ${ }^{(3,4)}$ The variation in nanoparticle size can be applied in the fields of solar cells, display technology, electrocatalysis, biosensors, and optical detection. For electrocatalysis, a high ratio of the number of surface atoms to the volume is provided by MNPs, which are used as heterogeneous catalysts, ${ }^{(6)}$ and MNPs can also enhance the effect of electron transmission by improving the defect space on the surface by thin-film engineering. ${ }^{(5)}$ In biosensors, the MNPs obtained from gold can provide a suitable microenvironment for bimolecular immobilization, retaining the biological activity of biomolecules and facilitating electron transfer between the immobilized proteins and the electrode surface. ${ }^{(6)}$ The effect of localized surface plasmon resonance (LSPR) on gold nanoparticles (GNPs) can clearly be observed by absorption to further realize the electron excitation of different MNPs. ${ }^{(7)}$ This principle can be applied in sensors. When nanoparticles are combined with an analyte, surface-enhanced Raman scattering, which is often used in optical detection, biochemical sensing, and disease treatment, can be observed.

On the other hand, the blending of water into an organic solvent (usually acetone) has always been preferential in developing an environmentally benign route for GNP preparation. ${ }^{(8)}$ For instance, hydrogen and hydroxyl bonds play a crucial role in aqueous organocatalytic aldol reactions, which are one of the most versatile and powerful tools for the construction of new $\mathrm{C}-\mathrm{C}$ bonds. Although the acetone added to the growth solution can effectively increase the yield of nanoparticles, the micelle template will be relaxed heterogeneously by the aldol condensation reaction, ${ }^{(9)}$ indicating the existence of nonuniform particles and self-assembly. Therefore, fruitful multidisciplinary collaborations between nanosciences and organic chemistry have developed into an expeditious and ever-increasing research subject corresponding to the remarkable catalytic activities and selectivity of nanometric materials. The physical qualities of nanoparticles are one of the most critical areas of study since the control of their size, shape, and surface crystallographic orientation ${ }^{(10)}$ by adding an organic solvent and an acid-base solution is a burgeoning field in electrical synthesis with sonochemistry.

Breakthroughs in achieving greater uniformity and smaller GNPs are required. In this study, we used electrochemical synthesis of a two-electrode system, including an electrolyte, a working electrode, and a counter/reference electrode, to fabricate GNPs as an alternative to conventional manufacturing involving chemical reduction, laser ablation, crystal planting, physical and chemical vapor deposition, and the sol-gel method. ${ }^{(11)}$ Although many factors affect the results, including the operating current, solution temperature, solution concentration and amount, ultrasonic oscillation time, and the immersed height and area of the electrode, the preparation of a growth solution can play an essential role in increasing the quality of GNPs. Therefore, the growth solution used is mixed with acetone and sodium hydroxide $(\mathrm{NaOH})$. The $\mathrm{NaOH}$ is used as a catalyst in the condensation reaction induced by acetone to effectively synthesize GNPs with uniform size and shape. An intense or sluggish condensation reaction will also be moderated because $\mathrm{NaOH}$ is useful for promoting the balance between the micelle template and the solution. Finally, the result not only reveals the effects of $\mathrm{NaOH}$ on GNPs, including the color of the solution, the full width at half maximum (FWHM), and the absorption wavelength, but also indicates the optimized parameters of electrochemistry preparation. We find that the observed change in the color of the GNP solution directly indicates the particle size, corresponding to the results of transmission electron microscopy (TEM); a red solution indicates large nanoparticles and a blue solution indicates small nanoparticles. 


\section{Experimental Process and Method}

\subsection{Materials}

(a) The growth solution was prepared from $0.08 \mathrm{M}$ cetyltrimethylammonium bromide (CTAB, Fluka, 98\%) as the powder species, $3 \mathrm{ml}$ of deionized water (DI water, 18.2 M 2 ), $0.1 \mathrm{~mol}$ of acetone $\left(\mathrm{CH}_{3} \mathrm{COCH}_{3}\right.$, J. T. Baker), and $0.1 \mathrm{~mol}$ of sodium hydroxide $(\mathrm{NaOH}$, Riedel-de Haën, Merck) in a test tube as the liquid species.

(b) Aqua regia (nitric acid hydrochloride) solution was prepared from hydrochloric acid ( $\mathrm{HCl}, \mathrm{J} . \mathrm{T}$. Baker, 37\%) and nitric acid $\left(\mathrm{HNO}_{3}\right.$, Honeywell Fluka) at a volume ratio of 1:3.

(c) Electrochemical synthesis with two electrodes is made up of a gold plate ( $\mathrm{Au}, 99 \%)$ and a platinum plate (Pt, 99\%) connected with solid conductors (copper wire, 99\%) to form an electrolyzer. A power supply (Agilent E3647A) provided a steady operating current through the fixed electrode with a fixed depth $(8 \mathrm{~mm})$ below the liquid level. Details of each material are also listed in Table 1.

\subsection{Synthesis of GNPs}

(a) Both $\mathrm{Au}$ and Pt were cut into suitable pieces $\left(30 \times 10 \times 0.5 \mathrm{~mm}^{3}\right)$ to act as the anode and cathode, respectively. After a standard cleaning operation including polishing with fine sandpaper, soaking in aqua regia solution, and washing in DI water for $5 \mathrm{~min}$ each, the two electrodes were dried with nitrogen gas, positioned $5 \mathrm{~mm}$ apart, and secured using Teflon spacers.

(b) Then, the test tube containing the growth solution was immersed in an ultrasonic oscillator (Hwashin, model 420) at $40 \mathrm{kHz}$ for 5 min with the water temperature held at $24 \pm 1{ }^{\circ} \mathrm{C}$. The electric voltage between two electrodes was set at $2.5 \mathrm{~V}$ when the contents of the test tube were electrolyzed and oscillated at $1 \mathrm{~mA}$ for $5 \mathrm{~min}$. The entire electrochemical synthesis lasted $5 \mathrm{~min}$ and each parameter is listed in Table 2.

(c) GNPs in powder form were obtained by the following steps: (i) the interface (GNPs, toluene, and surfactant from bottom to top) was separated by adding toluene to the product solution and then centrifuging at $12000 \mathrm{rpm}$ for $30 \mathrm{~min}$. (ii) After extracting the CTAB solution, which formed as a surfactant in the interlayer, GNPs were acquired through drying in an electric oven at $100{ }^{\circ} \mathrm{C}$ for three days.

Table 1

Detailed information of materials.

\begin{tabular}{lcc}
\hline Item & Value & Unit \\
\hline Au plate & $30 \times 10 \times 0.5$ & $\mathrm{~mm}$ \\
Pt plate & $30 \times 10 \times 0.5$ & $\mathrm{~mm}$ \\
CTAB powder $(0.08 \mathrm{M})$ & 87.5 & $\mathrm{mg}$ \\
DI water & 18.2 & $\mathrm{M} \Omega$ \\
Acetone & $300 / 500 / 700 / 900$ & $\mu \mathrm{l}$ \\
$\mathrm{NaOH}(0.1 \mathrm{~mol})$ & $30 / 50 / 70 / 90$ & $\mu 1$ \\
Aqua regia $\left(\mathrm{HNO}_{3}\right.$ per $\left.\mathrm{HCl}\right)$ & $1: 3$ & volume \\
\hline
\end{tabular}


Table 2

Parameters for electrochemistry fabrication.

\begin{tabular}{lcc}
\hline Parameter & Value & Unit \\
\hline Depth of electrode below liquid level & 8 & $\mathrm{~mm}$ \\
Oscillation frequency & 40 & $\mathrm{kHz}$ \\
Oscillation temperature & $24 \pm 1$ & ${ }^{\circ} \mathrm{C}$ \\
Operating current & 1 & $\mathrm{~mA}$ \\
Oscillation and operation time & 5 & $\mathrm{~min}$ \\
\hline
\end{tabular}

\subsection{Measurement and calculation of GNP characteristics}

The optical performance of GNP specimens in DI water in a quartz tube of $12.5 \times 12.5 \times$ $45 \mathrm{~mm}^{3}$ was measured by ultraviolet/visible (UV/Vis) spectroscopy (HITACHI, U-3900) in the spectral region from 350 to $850 \mathrm{~nm}$. The morphology of the microstructure was observed by analytical scanning TEM (JEM-3010, JEOL) at an acceleration voltage of $200 \mathrm{kV}$. The composition of GNPs was also obtained by energy-dispersive X-ray (EDX) spectroscopy. The GNP specimens used for TEM observation were prepared in three steps: mixing, centrifugation, and baking. The mixing solution, obtained by adding DI water or toluene, was centrifuged to extract the hierarchical CTAB solution. Both centrifugation and extraction were performed three times before the residue was placed on a standard copper grid (Formvar carbon supported film) and dried in an electric oven at $100{ }^{\circ} \mathrm{C}$.

\section{Results and Discussion}

A schematic diagram of the preparation of GNPs via electrochemical synthesis using the two-electrode system is shown in Fig. 1. The function of the ultrasonic oscillation process is to increase the production of hydroxyl groups in the growth solution. ${ }^{(12)}$ The hydroxide can cause more gold ions $\left(\mathrm{Au}^{+}\right)$to be reduced to atoms.

$$
\begin{gathered}
\mathrm{H}_{2} \mathrm{O} \rightarrow \mathrm{H}^{+}+\mathrm{OH}^{-} \text {(sonolysis) } \\
\mathrm{H}^{+}+\mathrm{RH}^{-} \rightarrow \mathrm{H}^{+}+\mathrm{H}_{2} \mathrm{O}
\end{gathered}
$$

From Eqs. (1) and (2), free radicals are determined to be $\mathrm{H}^{+}, \mathrm{OH}^{-}$, and $\mathrm{R}$ from the surfactant of $\mathrm{RH}$. By ultrasonic oscillation, $\mathrm{H}^{+}$and $\mathrm{OH}^{-}$are formed because the growth solution has produced cavitation [Eq. (1)]. Then, $\mathrm{R}$ free radicals are generated in the $\mathrm{RH}$ aqueous solution [Eq. (2)].

$$
\begin{gathered}
\mathrm{RH} \rightarrow \mathrm{R}+\mathrm{H}(\text { sonolysis }) \\
\mathrm{R}+\mathrm{Au}^{m+} \rightarrow \mathrm{Au}^{m-1}+\mathrm{H}^{+}+\mathrm{R}
\end{gathered}
$$




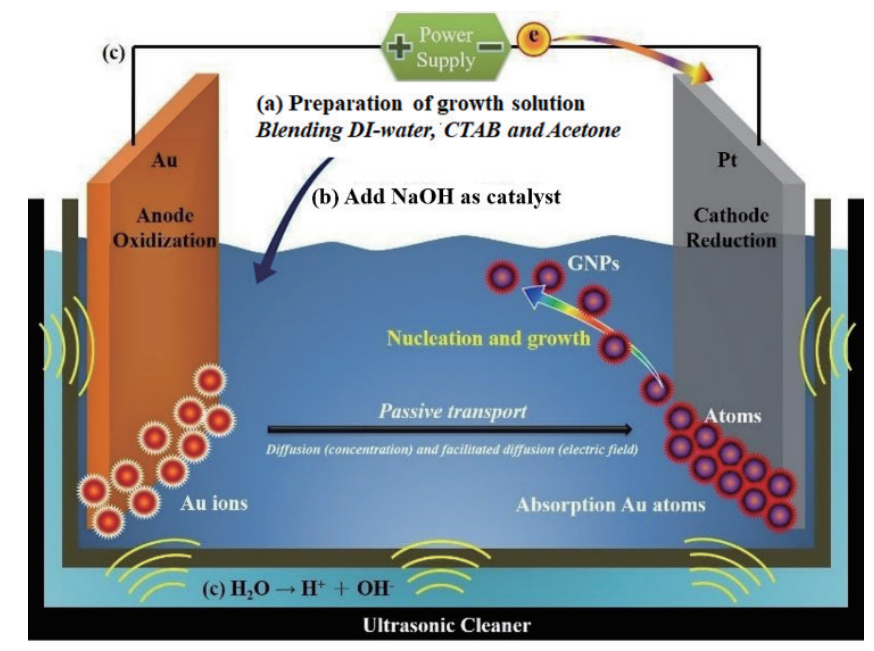

Fig. 1. (Color online) Schematic diagram of GNP preparation via electrochemistry: (a) prepare growth solution, (b) add $\mathrm{NaOH}$ as catalyst to form micelle template for condensation reaction, and (c) perform continuous electrolysis and oscillation.

By the redox reaction of free radicals and positive Au ions in Eq. (3), Au ions will gradually be reduced to a zero-valent metal [Eq. (4)] and the overall reduction rate will be increased. In addition, ultrasonic oscillation evenly disperses ions and micelles, and reduces ions in the vicinity of the platinum electrode. This decreases the probability that $\mathrm{Au}$ atoms are electroplated directly on the surface of the platinum electrode, thus increasing the reduction efficiency in the growth solution and the yield of GNPs.

The GNPs exhibit strong surface plasmon resonance (SPR) in the visible-to-near-infrared region, involving molecular vibration in the near-infrared region and molecular rotation in the microwave-to-infrared region. Moreover, the position and width of the plasmon band depend on the size, shape, and polydispersity of the GNPs. Figure 2 shows the light absorbance spectra of GNPs prepared by adding different amounts of acetone. The absorbance peaks of all samples are typical SPR peaks around 529.4, 526, 531, and $527.2 \mathrm{~nm}$ for the addition of 300, 500, 700, and $900 \mu \mathrm{l}$ acetone, respectively. The intensity of the absorbance peak gradually increases when the amount of acetone increases from 300 to $700 \mu$ l, then decreases. To determine the characteristics of the GNPs, however, the detailed relationship between the peak position and the particle size was studied in depth by assuming a surrounding isotropic matrix with the dielectric constant $\varepsilon_{m} .{ }^{(13)}$ The dielectric function $\varepsilon_{\infty}$ at high frequencies and the plasma wavelength of the bulk metal, $\lambda_{p}$, are the most important parameters, which are directly affected by the particle size and the concentration of free electrons in the metal. ${ }^{(14)}$ The variabilities of the size and distribution of GNPs are respectively obtained from the calculated FWHM and by TEM observation. ${ }^{(15,16)}$

Figure 3 shows the FWHM of the GNPs prepared from growth solutions with 300 to $900 \mu \mathrm{l}$ of added acetone. The addition of $700 \mu \mathrm{l}$ of acetone gave the maximum FWHM of $61 \mathrm{~nm}$, compared with values of 48 and $50 \mathrm{~nm}$ for the addition of 300 and $900 \mu \mathrm{l}$ of acetone, respectively. The minimum FWHM of $45.6 \mathrm{~nm}$ was obtained when $500 \mu \mathrm{l}$ of acetone was 


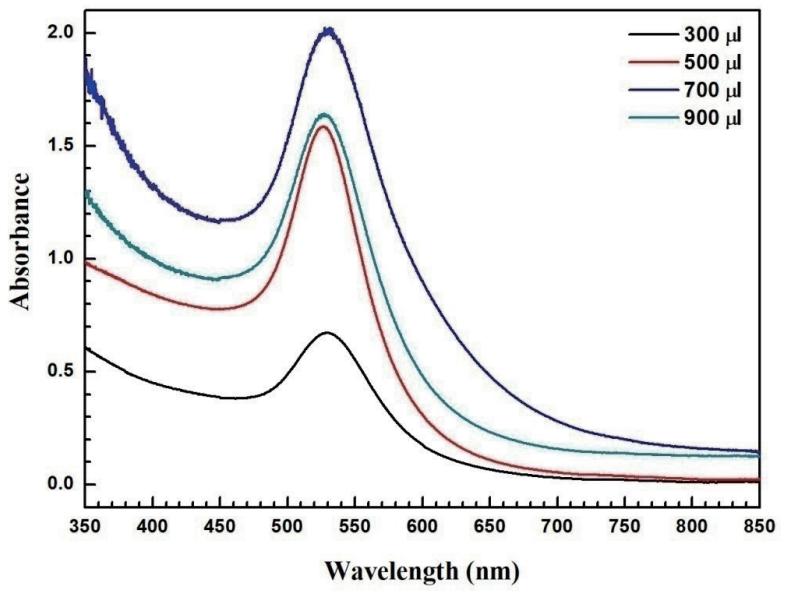

Fig. 2. (Color online) Light absorbance spectra of GNPs synthesized in growth solution with various amounts of added acetone.

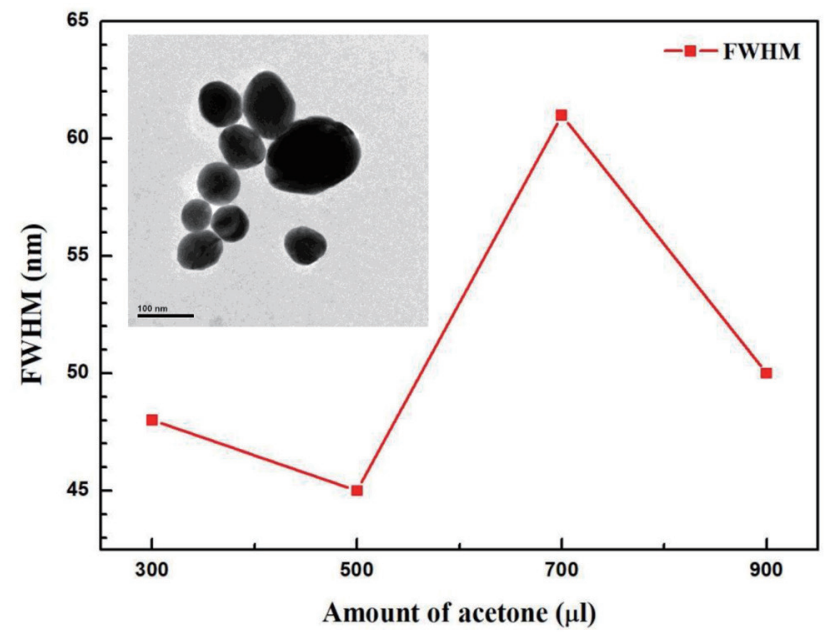

Fig. 3. (Color online) FWHM calculated from absorbance of GNP solutions with various amounts of added acetone. The inset shows a TEM image of GNPs added with $500 \mu$ l of acetone.

added. Both the FWHM calculated from the absorbance spectra and the SEM image effectively indicated the size distribution of the GNPs. A SEM image of the particles with the lowest FWHM of the absorbance spectra of $45.6 \mathrm{~nm}$ is shown as an inset in Fig. 3, which indicates that most of the GNPs are spherical with a size distribution of $50 \mathrm{~nm}$, although there are two much larger ellipsoidal particles with major axes of 115 and $175 \mathrm{~nm}$.

The purpose of adding acetone to the growth solution is to assist the formation of the micelle template. ${ }^{(17-19)}$ Also, acetone added to a surfactant can usually decompose the polar group of the ionic micelles at the interface between water hydrocarbons. ${ }^{(12)}$ This reduces the surface charge density of ionic micelles and causes electric double layers into resolving, thereby changing the geometry of the surfactant micelle template. The surface charge density is given by the Nernst equation 


$$
E=E_{0}+\left(R_{g} T / n_{i} F\right) \ln ^{a i}
$$

where $E$ is the electrode potential, $E_{0}$ is the standard electrode potential when the ion concentration is $1, n_{i}$ is the valence state of the ions, $R_{g}$ is the ideal gas constant, $T$ is the absolute temperature, $F$ is the Faraday constant, and $a i$ is the activity concentration. This equation clearly indicates that the surface potential of a solid varies with the concentration of ions in the ambient solution. The addition of acetone as an organic solvent during the electrolysis changes the shape of the micelle template. Another factor making the catalyst more susceptible to GNPs is the condensation reaction of acetone. The aldol condensation of ketones is an important reaction in industry, resulting in a $\beta$-hydroxy aldehyde or a $\beta$-hydroxy ketone from an addition reaction between two ketones. Subsequent dehydration produces an $\alpha, \beta$-unsaturated aldehyde or ketone. The addition step without subsequent dehydration is an aldol reaction given by Eqs. (6) and (7).

$$
\begin{gathered}
\mathrm{CH}_{3} \mathrm{COCH}_{3} \rightarrow \mathrm{CH}_{3} \mathrm{COCH}_{2} \mathrm{C}\left(\mathrm{CH}_{3}\right)_{2} \mathrm{OH} \\
\mathrm{CH}_{3} \mathrm{COCH}_{3} \rightarrow \mathrm{CH}_{3} \mathrm{COCHC}\left(\mathrm{CH}_{3}\right)_{2} \quad \text { Aldol reaction (6) } \\
\text { Aldol condensation (7) }
\end{gathered}
$$

The concentration of ions is increased by the extra hydroxyl group or pi bond from the resultant $\beta$-hydroxy ketone or $\alpha, \beta$-unsaturated ketone. The condensation reaction time is too long to control the size effectively via resultants, but both the aldol and dehydration steps may be promoted by $\mathrm{NaOH}$ solution.

Figure 4 shows the light absorbance spectra of GNPs prepared from the mixed growth solution obtained with $500 \mu \mathrm{l}$ of acetone and different amounts of added $\mathrm{NaOH}(30,50,70$, and $90 \mu 1)$. The absorbance peaks of all samples are typical SPR peaks around 530.6, 532.6, 527.4,

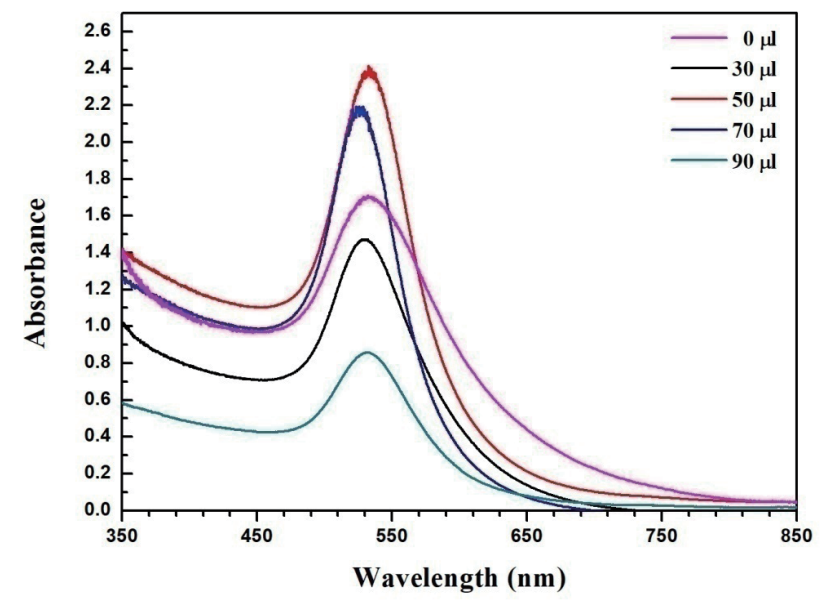

Fig. 4. (Color online) Light absorbance spectra of GNP solutions obtained by condensation reaction with different amounts of $\mathrm{NaOH}$ added to growth solution. 
and $532.8 \mathrm{~nm}$ for $30,50,70$, and $90 \mu 1$ of added $\mathrm{NaOH}$, respectively. Compared with the sample with $500 \mu \mathrm{l}$ of acetone alone $(0 \mu \mathrm{l}$ of $\mathrm{NaOH})$, the original peak position of $526 \mathrm{~nm}$ undergoes a slight blueshift. With increasing amount of added $\mathrm{NaOH}$, the absorbance of SPR first decreases but then increases to its maximum value with the addition of $50 \mu 1$ of $\mathrm{NaOH}$. Further increasing the amount of added $\mathrm{NaOH}$ to 70 and $90 \mu \mathrm{l}$ causes the intensity of SPR absorbance to decrease to less than that of the original sample with $0 \mu \mathrm{l}$ of $\mathrm{NaOH}$. It is clear that an appropriate amount of $\mathrm{NaOH}$ can effectively increase the SPR intensity of GNPs, although too much or too little $\mathrm{NaOH}$ solution reduces the SPR intensity of GNPs, leading to variations in their size and shape. Using $\mathrm{NaOH}$ results in the formation of uniform particles as observed by TEM in Fig. 5.

Figure 5 presents TEM images of GNPs synthesized with the condensation reaction and an improved micelle template with different amounts of $\mathrm{NaOH}(30,50,70$, and $90 \mu \mathrm{l})$ in the growth solution. Cluster agglomeration is clearly observed for the samples with 30 and $50 \mu 1$ of $\mathrm{NaOH}$ in Figs. 5(a) and 5(b), respectively, whereas there is no cluster agglomeration for the samples with 70 and $90 \mu \mathrm{l}$ of $\mathrm{NaOH}$, although the GNPs are slightly larger [Figs. 5(c) and 5(d)].

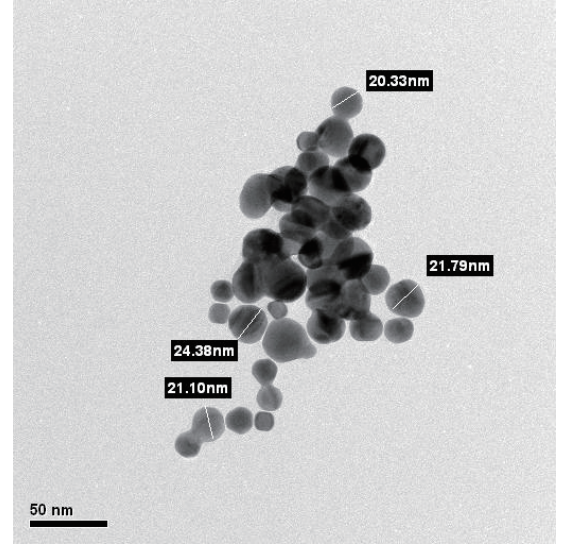

(a)

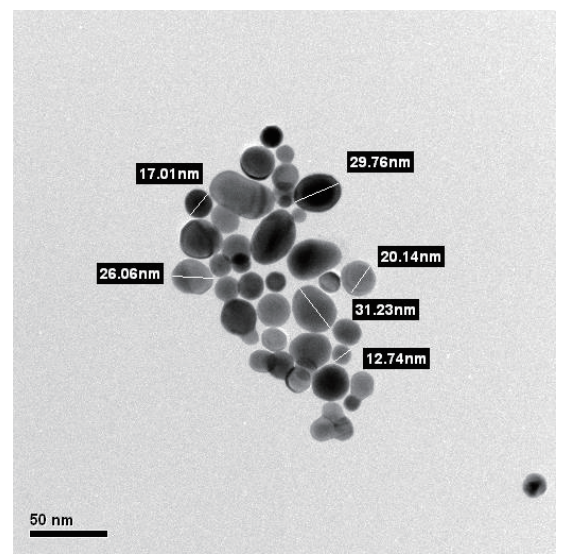

(c)

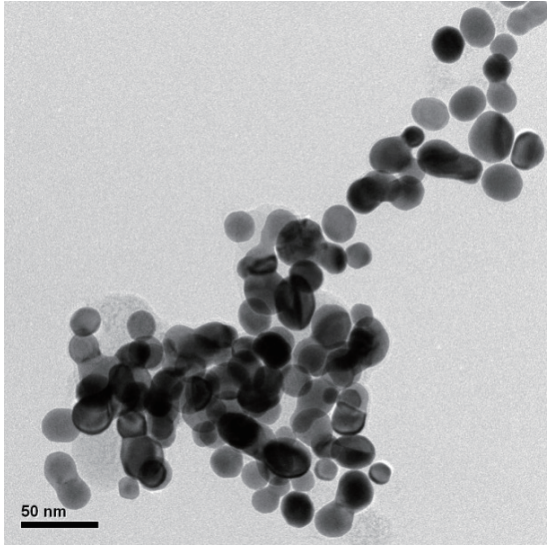

(b)

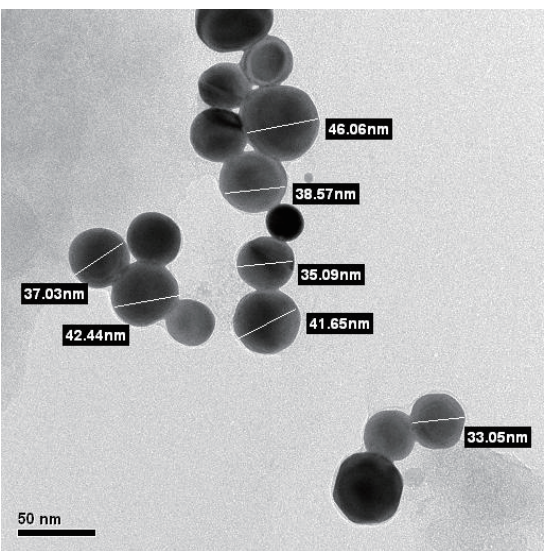

(d)

Fig. 5. TEM images of GNPs in condensation reaction obtained with (a) 30, (b) 50, (c) 70, and (d) $90 \mu 1$ of $\mathrm{NaOH}$ added to growth solution. 
In addition, the variation in the size of the GNPs is greatest when $90 \mu \mathrm{l}$ of $\mathrm{NaOH}$ is added. This is attributed to the strong pi bonds formed in the rapid condensation reaction and the hydroxyl groups from $\mathrm{NaOH}$, which provide a high ion concentration and greatly loosen the micelle template at the same time. Therefore, the uniformity and yield of the GNPs increase with a suitable amount of $\mathrm{NaOH}$ compared with those when using acetone alone. To calculate the size distribution, we estimated the proportion of the black area per total area counted by the 100 grid area ratio, which is shown in Fig. 6, and a diagram of the actual distribution is shown in Fig. 7.

Figure 6 shows a comparison of GNPs, including the above-mentioned proportion, average diameter, and FWHM. The proportions for 30, 50, 70, and $90 \mu \mathrm{l}$ of $\mathrm{NaOH}$ are similar, with values of $11,6.5,11.5$, and $11.5 \%$, and the average diameters are $29.8,25.2,25.5$, and $35 \mathrm{~nm}$, respectively. Too little or too much $\mathrm{NaOH}$ solution can cause either an ineffective or dramatic condensation reaction, and thus the minimum average diameter of GNPs is obtained by adding 50 and $70 \mu \mathrm{l}$ of $\mathrm{NaOH}$. However, we found that the smallest FWHM was obtained for $70 \mu 1$ of $\mathrm{NaOH}$. The small FWHM is related to the size distribution since the broadening of the absorption band is induced by the size dispersion of GNPs, resulting in the concentration of free electrons in the metal being sensitive to the particle size. ${ }^{(14)}$ That is, both the average size and the size distribution can affect the absorption band..$^{(7,14,20)}$ The interface of the micelle template is improved after the addition of $70 \mu \mathrm{l}$ of $\mathrm{NaOH}$ because the pi bonds formed by the rapid condensation reaction will expedite relaxation through the increased number of hydroxyl groups from $\mathrm{NaOH}$. The actual size distributions of the prepared GNPs are shown as histograms in Fig. 7, which correspond to the results shown in Fig. 6. The size distribution of the particles prepared with $70 \mu \mathrm{l}$ of $\mathrm{NaOH}$ is broader and more uniform than that of the particles prepared with $50 \mu 1$ of $\mathrm{NaOH}$. For the growth solutions prepared with 30 and $90 \mu \mathrm{l}$ of $\mathrm{NaOH}$, there are few GNPs with a small size below $10 \mathrm{~nm}$ owing to the low catalytic effect.

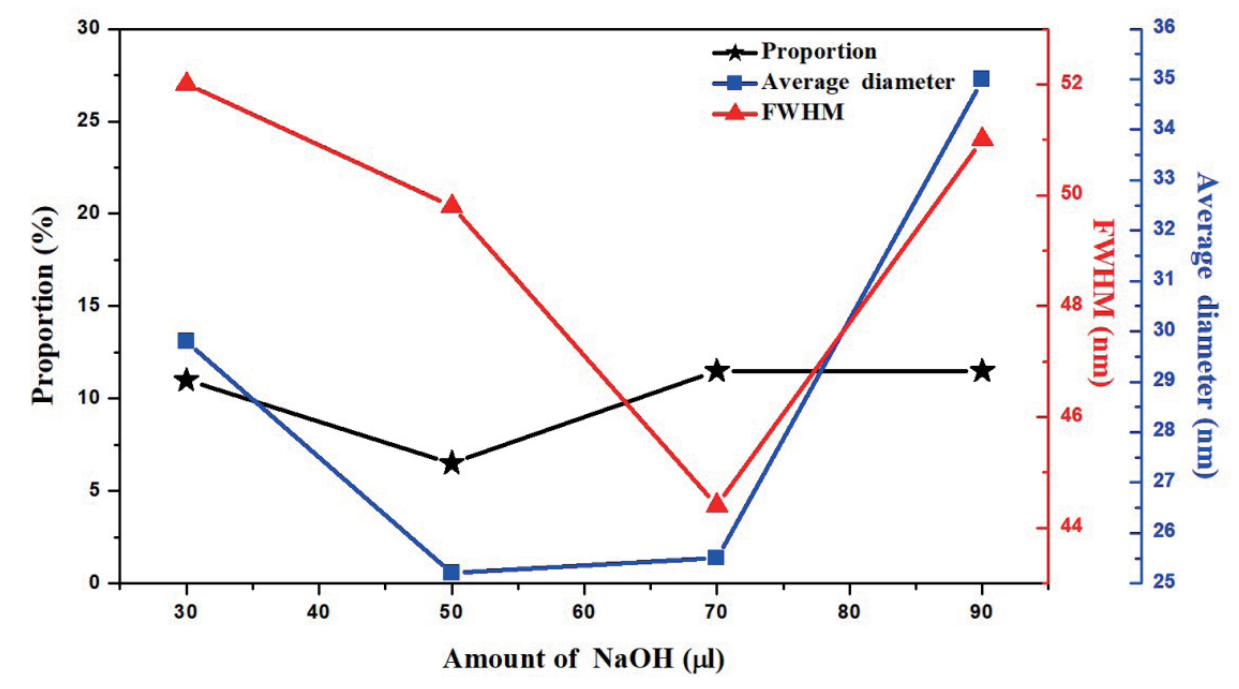

Fig. 6. (Color online) Calculated proportion of black area in images (stars), FWHM (triangles), and average diameter (squares) of GNPs obtained with $30,50,70$, and $90 \mu \mathrm{l}$ of $\mathrm{NaOH}$ added to growth solution. 


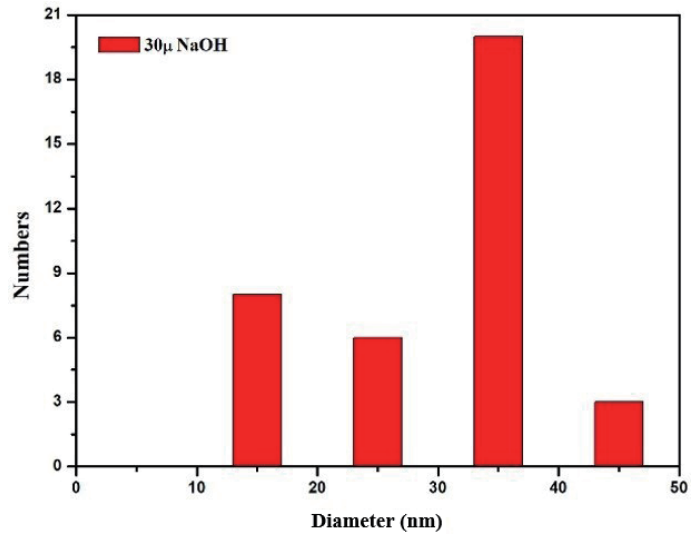

(a)

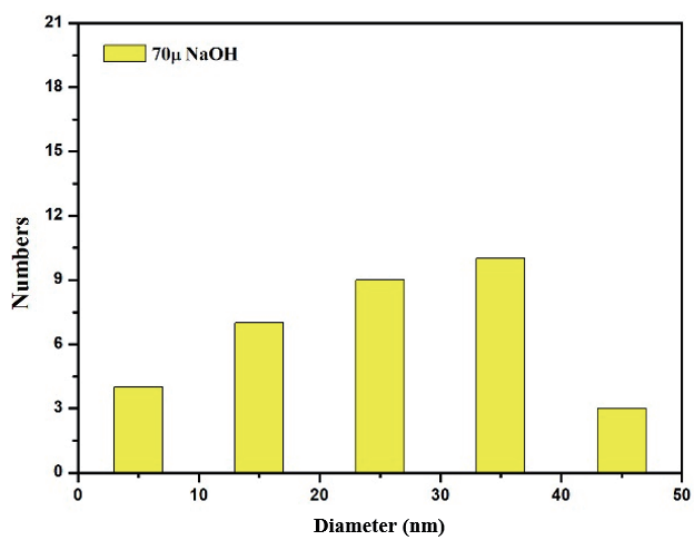

(c)

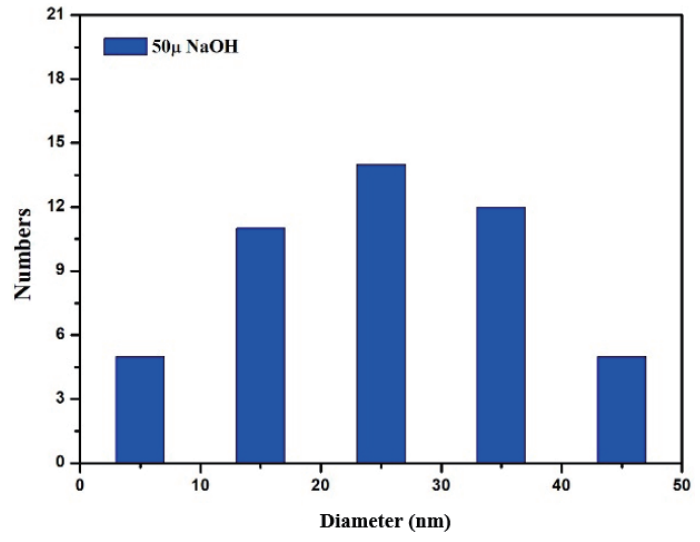

(b)

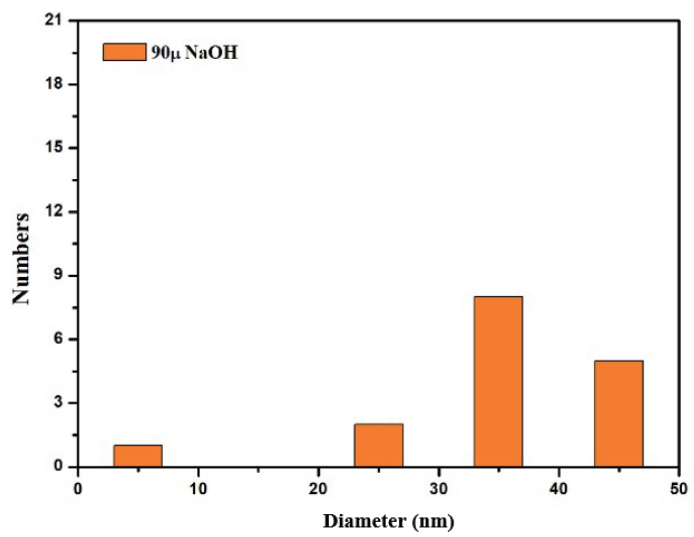

(d)

Fig. 7. (Color online) Histograms showing size distributions of GNPs obtained with 30, 50, 70 and $90 \mu 1$ of $\mathrm{NaOH}$ added to growth solution.

\section{Conclusion}

An innovative electrochemical synthesis with a two-electrode system was performed to effectively prepare GNPs with uniform size and shape through the addition of $\mathrm{NaOH}$ as a catalyst to the growth solution. The formula of the growth solution was improved by adding acetone, $\mathrm{CTAB}$, and $\mathrm{NaOH}$ simultaneously to control the micelle template because the condensation reaction from acetone is balanced via the hydroxyl groups from $\mathrm{NaOH}$. The main reason for the increased uniformity of GNPs was to maintain the steady expansion of the interface of the micelle template. Adding $70 \mu \mathrm{l}$ of $\mathrm{NaOH}$ to the growth solution with $500 \mu \mathrm{l}$ of acetone was more effective than using $500 \mu \mathrm{l}$ of acetone alone because the resultant growth solution boosted the ion concentration caused by hydroxyl groups and pi bonds. The absorbance spectra and TEM images also showed that the FWHM was smaller and the GNPs were more uniform for this mixed growth solution, indicating that GNPs with different sizes can be synthesized by using an appropriate amount of $\mathrm{NaOH}$ to tune the condensation reaction. As a 
result, GNPs with an average diameter of $25.5 \mathrm{~nm}$ were obtained, smaller than those in many studies. The above-mentioned small GNPs will be applied to various sensors or other devices.

\section{Acknowledgments}

This work was sponsored by the Ministry of Science and Technology (MOST) of the Republic of China under contract numbers 108-2221-E-390-006 and 109-2221-E-390-008.

\section{References}

1 S. S. Lucky, K. C. Soo, and Y. Zhang: Chem. Rev. 115 (2015) 1990. https://doi.org/10.1021/cr5004198

2 K. Ariga, J. Li, J. Fei, Q. Ji, and J. P. Hill: Adv. Mater. 28 (2016) 1251. https://doi.org/10.1002/adma.201502545

3 V. Malgras, Q. Ji, Y. Kamachi, T. Mori, F.-K. Shieh, K. C.-W. Wu, K. Ariga, and Y. Yamauchi: Bull. Chem. Soc. Jpn. 88 (2015) 1171. https://doi.org/10.1246/bcsj.20150143

4 O. S. Wolfbeis: Chem. Soc. Rev. 44 (2015) 4743. https://doi.org/10.1039/C4CS00392F

5 S. Linic, U. Aslam, C. Boerigter, and M. Morabito: Nat. Mater. 14 (2015) 567. https://doi.org/10.1038/nmat4281

6 C. Li, W. Cai, B. Cao, F. Sun, Y. Li, C. Kan, and L. Zhang: Adv. Funct. Mater. 16 (2006) 83. https://doi. org/10.1002/adfm.200500209

7 C. D. Medley, J. E. Smith, Z. Tang, Y. Wu, S. Bamrungsap, and W. Tan: Anal. Chem. 80 (2008) 1067. https:// doi.org/10.1021/ac702037y

8 A. R. Vilchis-Nestor, V. Sánchez-Mendieta, M. A. Camacho-López, R. M. Gómez-Espinosa, M. A. CamachoLópez, and J. A. Arenas-Alatorre: Mater. Lett. 62 (2008) 3103. https://doi.org/10.1155/2017/8108504

9 C. Li, F. Fan, B. Yin, L. Chen, T. Ganguly, and Z. Tian: Nano Res. 6 (2013) 29. https://doi.org/10.1007/s12274012-0278-2

10 Y. Pan, S. Neuss, A. Leifert, M. Fischler, F. Wen, U. Simon, G. Schmid, W. Brandau, and W. Jahnen-Dechent: Small 3 (2007) 1941. https://doi.org/10.1002/smll.200700378

11 M.-C. Daniel and D. Astruc: Chem. Rev. 104 (2004) 293. https://doi.org/10.1021/cr030698+

12 G. Mie: Annalen der physik 330 (1908) 377. https://doi.org/10.1002/andp.19083300302

13 P. Mulvaney, L. Liz-Marzan, M. Giersig, and T. Ung: J. Mater. Chem. 10 (2000) 1259. https://doi.org/10.1039/ B000136H

14 M. Kawasaki and K. Masuda: J. Phys. Chem. B 109 (2005) 9379. https://doi.org/10.1021/jp0442044

15 P. K. Jain, K. S. Lee, I. H. El-Sayed, and M. A. El-Sayed: J. Phys. Chem. B 110 (2006) 7238. https://doi. org/10.1021/jp057170o

16 P. Galletto, P. Brevet, H. Girault, R. Antoine, and M. Broyer: J. Phys. Chem. B 103 (1999) 8706. https://doi. org/10.1021/jp991937t

17 S.-S. Chang, C.-W. Shih, C.-D. Chen, W.-C. Lai, and C. C. Wang: Langmuir 15 (1999) 701. https://doi. org/10.1021/jp971656q

18 M. Törnblom and U. Henriksson: J. Phys. Chem. B 101 (1997) 6028-6035. https://doi.org/10.1021/jp970899f

19 Y. Mizukoshi, K. Okitsu, Y. Maeda, T. A. Yamamoto, R. Oshima, and Y. Nagata: J. Phys. Chem. B 101 (1997) 7033. https://doi.org/10.1021/jp9638090

20 A. S. Rubio: Modified Au-Based Nanomaterials Studied by Surface Plasmon Resonance Spectroscopy (Springer, 2015). https://doi.org/10.1007/978-3-319-19402-8 